\title{
Trattamento dell'iperparatiroidismo
} secondario: calciomimetici, attivatori selettivi del recettore della vitamina D (VDRAs) o terapia combinata?

\author{
M. Cozzolino, S. Pasho, C. Crovetto, E. Missaglia \\ U.O. Nefrologia e Dialisi, Azienda Ospedaliera San Paolo, Polo Università degli Studi di Milano, Milano
}

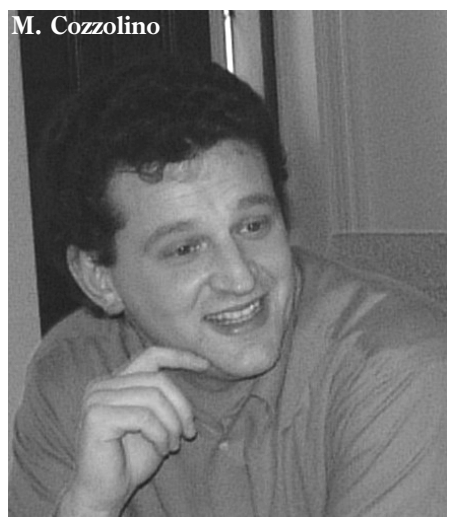

\section{Introduzione}

Una delle complicanze più frequenti in corso di insufficienza renale cronica (IRC) è rappresentata dall'iperparatiroidismo secondario (IPTS), caratterizzato da iperplasia delle ghiandole paratiroidee e da aumentata sintesi e secrezione di PTH (1-4). L'incremento dei livelli sierici di PTH è comunemente causa di danno a carico del tessuto osseo (osteodistrofia renale). Recentemente, è stato ben evidenziato che l'IPTS è causa di una tossicità sistemica, in particolar modo a livello dell'apparato cardiovascolare, aumentando il rischio di morbilità e di mortalità per causa cardiovascolare nei pazienti in dialisi (5-7). Da queste considerazioni nasce l'interesse per lo studio di nuove terapie che possano ridurre i livelli di PTH e di conseguenza controllare l'alterato metabolismo minerale in corso di IRC.

Nei soggetti con funzione renale normale, il tessuto paratiroideo non prolifera e rimane in fase quiescente (fase G0 del ciclo cellulare). In corso di IRC, l'ipocalcemia, l'iperfosforemia e il deficit di $1,25(\mathrm{OH})_{2} \mathrm{D}_{3}$ sono i tre fattori direttamente coinvolti nel determinare l'IPTS (Fig. 1). Liperfosforemia e il deficit di $1,25(\mathrm{OH})_{2} \mathrm{D}_{3}$, in parte indirettamente attraverso l'ipocalcemia, stimolano le cellule paratiroidee a proliferare e a secernere più PTH (8), attraverso meccanismi sia trascrizionali che post-trascri- zionali. Infatti, il calcio e $\mathrm{l}^{\prime} 1,25(\mathrm{OH})_{2} \mathrm{D}_{3}$ regolano la trascrizione del gene del PTH riducendo i propri recettori presenti sulle ghiandole paratiroidee, rispettivamente $\mathrm{i}$ Calcium Sensing Receptor (CaSR) e i Vitamin D Receptor (VDR) $(9,10)$. La riduzione del contenuto paratiroideo dei CaSR e dei VDR rende le ghiandole paratiroidee resistenti alla soppressione da parte del calcio e dell' $1,25(\mathrm{OH})_{2} \mathrm{D}_{3}$ della secrezione di PTH (9). Il calcio e il fosforo controllano la sintesi di PTH a livello post-trascrizionale, attraverso un legame con proteine citosoliche del m-RNA del $\mathrm{PTH}$, regolandone così la trascrizione stessa (11).

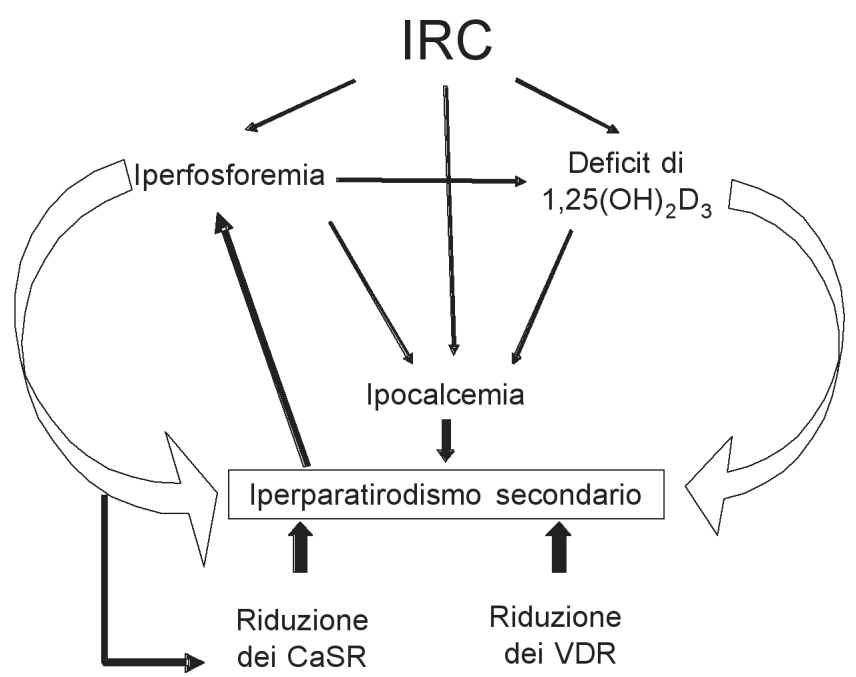

Fig. 1 - Meccanismi patogenetici dell'iperparatiroidismo secondario. 
È altresì noto come variazioni dei livelli sierici di calcio, fosforo, e di $1,25(\mathrm{OH})_{2} \mathrm{D}_{3}$ e contenuto paratiroideo di CaSR e VDR abbiano anche un impatto drammatico sulla crescita del tessuto paratiroideo (1-3). Recentemente, sono stati considerati nuovi meccanismi molecolari alla base dell'eziopatogenesi dell'IPTS in corso di IRC, in special modo sulla regolazione dell'iperplasia paratiroidea da parte del calcio, del fosforo e dell' $1,25(\mathrm{OH})_{2} \mathrm{D}_{3}$.

Lincremento dei livelli sierici di PTH è responsabile di una serie di conseguenze cliniche a lungo termine il cui target primario è lo scheletro. Oltre a ciò, si aggiunge una tossicità sistemica dell'IPTS a carico dell'apparato cardiovascolare, che spiega l'aumentato rischio di morbilità e mortalità cardiovascolare dei pazienti uremici. Ganesh et al (12) hanno dimostrato in un'ampia coorte di emodializzati un'associazione tra livelli di PTH >495 $\mathrm{pg} / \mathrm{mL}$ e un aumentato rischio relativo di morte improvvisa per causa cardiovascolare $(\mathrm{p}<0.05-\mathrm{RR}: 1.06)$, rispetto a un gruppo di controllo con livelli inferiori di PTH (197$495 \mathrm{pg} / \mathrm{mL}$ ). Il possibile ruolo del PTH nel determinare gli out-comes cardiovascolari nei pazienti in dialisi è stato inoltre valutato in un più recente studio prospettico europeo della durata di 6 anni. È stato dimostrato che livelli sierici di PTH $>471 \mathrm{pg} / \mathrm{mL}$ sono associati a un più elevato rischio di morte cardiovascolare rispetto a livelli di PTH inferiori (RR:3.9) (13).

Nonostante ciò, il PTH non può essere considerato l'unico attore metabolico coinvolto nel determinare la morte cardiovascolare dei pazienti uremici. Per esempio è stato evidenziato che pazienti con fosforemie superiori a $6.5 \mathrm{mg} / \mathrm{dL}$ hanno un rischio di morte che è del $41 \% \mathrm{su}-$ periore rispetto ai pazienti con livelli sierici di fosforo compresi tra 2.4 e $6.5 \mathrm{mg} / \mathrm{dL}$ (12). Dati simili sono stati descritti in ampie popolazioni di dializzati anche per quel che riguarda l'associazione tra elevati livelli sierici di calcio e mortalità cardiovascolare (14).

I meccanismi che sono alla base dell'aumentata morta- lità cardiovascolare dei pazienti uremici non sono noti, tuttavia si può assumere che l'imputato principale sia la deposizione di calcio a livello vascolare, che è stato identificato come un sicuro predittore di mortalità (15). Da tutto ciò si evince che l'individuazione dei livelli ottimali di PTH, fosforo e calcio sierici sia da considerare la base per scelte terapeutiche appropriate.

\section{Terapia dell'iperparatiroidismo secondario: il ruolo dei calciomimetici}

L'attuale terapia dell'IPTS del paziente uremico ha un successo limitato nell'ottenere il raggiungimento dei valori di PTH, calcio e fosforo indicati dalle Linee Guida internazionali (NKF-K/DOQI) (16) e nazionali (Società Italiana di Nefrologia) (17) (Tab. I).

I calciomimetici sono farmaci capaci di attivare il recettore sensibile al calcio, Calcium Sensing Receptor (CaR). La loro azione aumenta la sensibilità del CaR al calcio extracellulare, attivando il recettore a più bassi livelli di calcio. Il CaR è espresso a livello di molti organi e apparati, ma in particolare nelle cellule paratiroidee e in quelle tubulari renali. La sua attivazione induce una pronta riduzione della secrezione del PTH e, a livello renale, un aumento della calciuria. I calciomimetici si sono dimostrati nelle esperienze precliniche efficaci nel ridurre i livelli di PTH, prevenendo lo sviluppo dell'iperplasia ghiandolare e inducendo la regressione dell'osteite fibrosa nel modello sperimentale del ratto uremico (18). Il primo, e per ora, unico, calciomimetico impiegato nella terapia del SHPT è il cinacalcet-HCl. Questo composto, sperimentato in studi pre-clinici con il nome di AMG 073, ha dato buoni risultati in studi clinici di fase II (19-21) e fase III (21-22). È stato arruolato un gran numero di pazienti in dialisi, secondo un disegno di studio e con criteri di arruolamento sostanzialmente simili. Il cinacalcet è più efficace del placebo nel controllo dell'IPTS. A dosi

TABELLA I - RANGE DI RIFERIMENTO PER I LIVELLI DI PTH, P, Ca E CaxP INDICATI NELLE KDOQI GUIDELINES DEL 2003 E NELLE LINEE GUIDA DELLA SOCIETÀ ITALIANA DI NEFROLOGIA DEL 2007

\begin{tabular}{llllll}
\hline Stadio IRC & $\begin{array}{l}\text { Range } \\
\text { GFR } \\
(\mathrm{mL} / \mathrm{min})\end{array}$ & $\begin{array}{l}\text { IPTH } \\
(\mathrm{pg} / \mathrm{mL})\end{array}$ & $\begin{array}{l}\mathrm{P} \\
(\mathrm{mg} / \mathrm{dL})\end{array}$ & $\begin{array}{l}\mathrm{Ca} \\
(\mathrm{mg} / \mathrm{dL})\end{array}$ & $\begin{array}{l}\text { CaxP } \\
\left(\mathrm{mg}^{2} / \mathrm{dL}^{2}\right)\end{array}$ \\
\hline 3 & $30-59$ & $35-70$ & $2.7-4.6$ & Valori normali* & $<55$ \\
4 & $15-29$ & $70-110$ & $2.7-4.6$ & Valori normali* & $<55$ \\
5 & $<15$ o dialisi & $150-300$ & $3.5-5.5$ & $8.4-9.5$ & $<55$
\end{tabular}

$\left.{ }^{*}\right)$ Valori normali, corretti, del laboratorio di riferimento 
comprese fra $30-120 \mathrm{mg} /$ die, in unica somministrazione orale, riduce il PTH in media del $30-40 \%$ rispetto al basale, consentendo di portarne i livelli entro il target di $300 \mathrm{pg} / \mathrm{mL}$ in oltre il $50 \%$ dei pazienti. Tuttavia la quota di questi ultimi si riduce con l'aumentare dei livelli basali di PTH. La riduzione del PTH è dose-dipendente. A questa corrisponde una riduzione dei livelli di calcemia e di prodotto calcio-fosforo. L'associazione con chelanti del fosforo e con metabolici della vitamina $\mathrm{D}$ non influenza l'efficacia di cinacalcet, ma cinacalcet può comportare un maggior fabbisogno di chelanti a base di calcio. Gli effetti collaterali più significativi sono nausea e vomito, più frequenti nei pazienti in cinacalcet rispetto a placebo. Non disponiamo a oggi di dati sufficienti circa il suo profilo di efficacia/sicurezza nei pazienti con insufficienza renale cronica pre-dialitica, e in quelli con iperparatiroidismo persistente post-trapianto. Occorre un follow-up di più lungo periodo per stabilire i vantaggi di cinacalcet rispetto a incidenza di paratiroidectomie, fratture, patologia cardiovascolare.

Il calciomimetico può essere utilizzato in monoterapia soltanto in quei pazienti con iperparatiroidismo terziario e quindi ipercalcemici, per i quali rappresenta l'unica opzione terapeutica oltre alla paratiroidectomia chirurgica (24-27). Un dato di grande rilievo in questi soggetti, che rende l'uso del farmaco particolarmente maneggevole nella terapia dell'iperparatiroidismo terziario, è la contemporanea riduzione della calcemia e della fosforemia. In conclusione, gli studi clinici, che hanno prevalentemente utilizzato l'AMG 073 (cinacalcet), hanno confermato l'efficacia del farmaco nel controllo dell'IPTS dell'uremia. In particolare, in quest'ultima condizione il cinacalcet ha dimostrato un buon profilo di efficacia, potendo ridurre i livelli di PTH in associazione a una riduzione del calcio e parzialmente del fosforo (19). Nel circa $30 \%$ dei pazienti in terapia con cinacalcet gli effetti collaterali riportati sono riferibili a disturbi gastroenterici e ipocalcemia, solo raramente sintomatica. Gli effetti collaterali fin qui osservati sono soprattutto a carico dell'apparato gastroenterico, con una incidenza di nausea (32\% vs $19 \%$ ) e vomito (30\% vs $16 \%$ ) più elevati rispetto a placebo (19). Nausea e vomito colpivano il $34 \%$ e $44 \%$ dei pazienti nello studio di lungo periodo (21).

\section{Terapia dell'iperparatiroidismo secondario: il ruolo degli attivatori selettivi del recettore della vitamina D (VDRAs)}

L'attivazione dei recettori della vitamina $\mathrm{D}$ (VDR) è essenziale per il controllo della sintesi del PTH e della proliferazione delle cellule paratiroidee (1). Ciò diventa evidente quando si riduce l'espressione dei VDR a livello paratiroideo, conseguenza diretta della down-regulation e dei bassi livelli di vitamina D circolanti osservati nell'insufficienza renale (23). In studi sperimentali su animali uremici, è stato recentemente dimostrato come il calcitriolo e l'attivatore selettivo del VDR, paricalcitolo, siano in grado di controllare la proliferazione delle cellule paratiroidee attraverso l'inibizione di specifici fattori di crescita, quali il Transforming Growth Factor alfa e, il suo recettore, l'Epidermal Growth Factor Receptor, o attraverso la stimolazione di proteine che controllano il ciclo cellulare, come la p21, anche quando i ratti vengono nutriti con diete ad alto contenuto di fosforo. Un altro effetto positivo della somministrazione di vitamina $\mathrm{D}$ sui ratti uremici è l'up-regulation della sintesi dei CaSR sulla superficie delle cellule paratiroidee (8), una condizione che facilita la soppressione della secrezione di PTH da parte del calcio, spostando la curva Ca-PTH verso sinistra. Questo rende le cellule paratiroidee maggiormente sensibili alle concentrazioni del calcio extracellulare. Inoltre, è stato recentemente ipotizzato come l'attivazione selettiva dei VDR possa ridurre direttamente le calcificazioni cardiovascolari attraverso l'inibizione della produzione di proteine che promuovono tale processo o attraverso lo stimolo sull'espressione di proteine che inibiscono le calcificazioni vascolari. Se si considera che i VDR sono localizzati a livello della tunica media delle cellule muscolari lisce e che la somministrazione di calcitriolo alle cellule osteoblastiche è in grado di ridurre la sintesi del collagene tipo I, è facile comprendere come questo processo sarebbe inoltre capace di inibire la deposizione di calcio nella matrice extracellulare (24).

La scoperta dell'1,25-diidrossivitamina D3 (calcitriolo) aprì una nuova era nel trattamento dell'IPTS nei pazienti uremici a causa della sua selettiva e potente capacità di legame ai VDR rispetto al suo precursore, la 25-idrossivitamina D. Successivamente a questa prima generazione, fu il momento di una seconda generazione di composti in cui il gruppo idrossilico in posizione $25 \mathrm{fu}$ rimosso; tra questi ci sono la 1a-idrossivitamina $\mathrm{D}_{3}$ (alfacalcidiolo) e la 1a-idrossivitamina $\mathrm{D}_{2}$ (doxecalciferolo). Essi sono considerati pro-ormoni poiché richiedono una idrossilazione in posizione 25 a livello epatico (25).

La terza generazione di composti è rappresentata essenzialmente dal 19-nor-1,25-diidrossivitamina $\mathrm{D}_{2}$ (paricalcitolo) e dal 22-oxacalcitriolo (maxixalcitolo), entrambi con minor effetti sulla calcemia e sulla fosforemia rispetto al calcitriolo (26). Questi composti hanno suscitato notevole interesse perché hanno aperto la strada verso un trattamento più sicuro dell'IPTS a causa di un più ridotto numero di eventi di ipercalcemia. Infatti, è stata recentemente dimostrata una minore incidenza di ipercalcemia e iperfosforemia in ratti uremici trattati con il 
paricalcitolo rispetto al doxecalciferolo, verosimilmente a causa di un minore assorbimento intestinale di calcio e fosforo (27).

L'acquisizione di un'adeguata strategia e del giusto timing per il trattamento dell'IPTS nei pazienti con IRC diventa di cruciale importanza.

Mentre la seconda generazione di analoghi non ha destato molto interesse, verosimilmente a causa della necessità di un'idrossilazione in posizione 25 per una completa attivazione, la terza generazione di composti, in particolare il paricalcitolo, è stata a fondo analizzata per i suoi ridotti effetti sul calcio e sul fosfato sierici rispetto al calcitriolo $(26,27)$. Molti studi mostrano come l'1,25-diidrossi-19-nor-vitamina $\mathrm{D}_{2}$ (paricalcitolo) sia in grado nei modelli animali di controllare il PTH in assenza di ipercalcemia (26) e questi dati sono confermati da osservazioni cliniche (28).

Un confronto testa a testa tra paricalcitolo e calcitriolo è stato condotto da Sprague et al che hanno dimostrato in uno studio prospettico randomizzato e controllato che la somministrazione di paricalcitolo permette di ottenere una soppressione del $50 \%$ dei livelli di PTH, tre settimane prima rispetto al calcitriolo con un numero di episodi di ipercalcemia significativamente inferiori (29). Tutti questi dati suggeriscono che il paricalcitolo rappresenta un reale passo avanti nel controllo della secrezione del PTH nei pazienti uremici affetti da IPTS.

Uno studio epidemiologico condotto su un'ampia coorte di dializzati degli Stati Uniti ha recentemente mostrato che la terapia con paricalcitolo è associata a un beneficio sulla sopravvivenza del $16.6 \%$ dopo 3 anni di osservazione confrontato con la terapia con calcitriolo (30). Gli effetti benefici del paricalcitolo sono stati inoltre confermati nel sottogruppo di pazienti che sono passati dal calcitriolo al paricalcitolo; gli effetti positivi nel gruppo del paricalcitolo non hanno alcuna relazione con i quintili di calcemia, fosforemia e PTH (30).

In un secondo studio retrospettivo gli stessi autori hanno mostrato come i pazienti che ricevono qualsiasi tipo di VDRAs endovena (calcitriolo o paricalcitolo) abbiano una ridotta mortalità per tutte le cause, così come una ridotta mortalità cardiovascolare (del 50\% inferiore) rispetto ai pazienti che non ricevono vitamina $\mathrm{D}$ endovena nello stesso periodo di osservazione di 2 anni (31).

Più recentemente Dobrez et al (32) hanno mostrato in uno studio retrospettivo che i dializzati trattati con paricalcitolo vanno incontro anche a una minore morbilità, a una minore ospedalizzazione e a migliori out-comes, rispetto ai pazienti trattati con calcitriolo. Da tutto ciò si può concludere che per i pazienti in dialisi esistono differenti "profili di rischio" in relazione alla terapia con vitamina D: la prognosi peggiore è detenuta da quei pazienti che non sono trattati con alcun tipo di vitamina $\mathrm{D}$, la prognosi intermedia riguarda coloro che sono trattati con calcitriolo, la prognosi migliore quelli che ricevono in terapia paricalcitolo.

Considerati insieme questi ampi studi, sebbene retrospettivi, suggeriscono che la terapia pulsatile con i VDRAs è in grado di ridurre gli effetti deleteri che l'iperfosforemia, l'ipercalcemia e l'elevato PTH hanno sugli out-comes dei pazienti. Inoltre, se si considera che più del $50 \%$ delle morti tra i pazienti dializzati sono da attribuire a causa cardiovascolare, si può assumere che la terapia con vitamina $\mathrm{D}$ possa intervenire nei meccanismi coinvolti nell'invecchiamento vascolare.

\section{Conclusioni}

È parere diffuso che il cinacalcet e il paricalcitolo rappresentino due armi terapeutiche per il nefrologo per la prevenzione e il trattamento dell'IPTS e delle sue complicanze cardiovascolari. Una monoterapia con l'uno o con altro diventa sicuramente la scelta iniziale a seconda dello stato metabolico minerale del singolo paziente. Una terapia combinata diventa di seconda scelta, soprattutto per i problemi correlati al costo della stessa.

I pazienti con IPTS e livelli di calcemia ridotti o normali, e di fosforemia normali si giovano sicuramente del trattamento con VDRAs, sia per prevenire l'ulteriore aumento dei livelli di PTH sia per gli effetti "pleitropici" della vitamina $\mathrm{D}$ stessa. D'altra parte, il vantaggio maggiore della terapia con cinacalcet è la possibilità di controllare i livelli di PTH in quei pazienti con valori elevati calcio e fosforo, per poi poter introdurre in un secondo momento la terapia con VDRAs. Il limite più importante della terapia affidata ai calciomimetici è che essa non costituisce una correzione patogenetica della patologia osteo-metabolica dell'IPTS e deve pertanto essere affiancata obbligatoriamente dalle terapie che agiscono in modo correttivo su quelli che sono gli elementi etio-patogenetici certi (deficit e resistenza alla vitamina $\mathrm{D}$, bilancio positivo del fosforo, bilancio negativo calcico). L'uso dei calciomimetici, che rappresenta comunque un importante avanzamento nel campo della terapia dell'IPS dell'uremia, dovrà inoltre essere oggetto di uno stretto controllo clinico per le potenziali interferenze con altri sistemi cellulari dove le sue funzioni sono ancora poco note. Il calciomimetico infatti non deve sostituire il trattamento con vitamina D ma implementarlo: l'associazione dei calciomimetici con i metaboliti attivi della vitamina $\mathrm{D}$ di ultima generazione (paricalcitolo), permetterà una migliore soppressione del $\mathrm{PTH}$ anche a livello genomico, con riduzione drastica dei rischi di ipercalcemia e di iperfosforemia. 
Indirizzo degli Autori:

Dr. Mario Cozzolino

U.O. Nefrologia e Dialisi, AO San Paolo

Cattedra di Nefrologia, Università di Milano

Via A. di Rudini, 8

20142 Milano

mariocozzolino@hotmail.com

\section{Bibliografia}

11. Parfitt AM. The hyperparathyroidism of chronic renal failure: A disorder of growth. Kidney Int 1997; 52: 3-9.

2. Cozzolino M, Butti A, Chiarelli G, et al. New insights in the pathogenesis of secondary hyperparathyroidsm. G Ital Nefrol 2005; 4: 329-36.

3. Slatopolsky E, Finch J, Denda M, et al. Phosphorus restriction prevents parathyroid gland growth. High phosphorus directly stimulates PTH secretion in vitro. J Clin Invest 1996; 97: 2534-40.

4. Silver J, Bar Sela S, and Naveh-Many T. Regulation of parathyroid cell proliferation. Curr Op Nephrol Hyperten 1997; 6: 321-26.

5. Cozzolino M, Dusso A, Slatopolsky E. Role of calcium x phosphate product and bone associated proteins on vascular calcification in renal failure. J Am Soc Nephrol 2001; 12: 2511-6.

6. Cozzolino M, Brancaccio D, Gallieni M, and E. Slatopolsky. Pathogenesis of vascular calcification in chronic kidney disease. Kidney Int 2005; 68: 429-36.

7. Cozzolino M, Gallieni M, Brancaccio D, et al. Vitamin D retains an important role in the pathogenesis and management of secondary hyperparathyroidism in chronic renal failure. J Nephrol 2006; 19: 566-57.

8. Cozzolino M, Brancaccio D, Gallieni M, et al. Pathogenesis of parathyroid hyperplasia in renal failure. J Nephrol 2005; 18 (1): 5-8.

9. Brown EM, Gamba G, Riccardi D, et al. Cloning and characterization of an extracellular $\mathrm{Ca}^{2+}$-sensing receptor from bovine parathyroid. Nature 1993; 366: 575-80.

10. Merke J, Hugel U, Zlotkowski A, et al. Diminished parathyroid 1,25-dihydroxyvitman D3 receptors in experimental uremia. Kidney Int 1987; 32: 350-33.

11. Silver J, Kilav R, Naveh-Many T. Mechanisms of secondary hyperparathyroidism. Am J Physiol Ren Physiol 2002; 283: F367-76.
12. Ganesh SK, Stack AG, Levin NW, et al. Association of elevated serum $\mathrm{PO}_{4}, \mathrm{Ca} \times \mathrm{PO}_{4}$ product, and parathyroid hormone with cardiac mortality risk in chronic hemodialysis patients. J Am Soc Nephrol 2001; 12: 2131-8.

13. Marco MP, Craver L, Bertriu A, et al. Higher impact of mineral metabolism on cardiovascular mortality in an European hemodialysis population. Kidney Int 2003; 63 (Suppl 85): S111-4.

14. Block GA, Klassen PS, Lazarus JM, et al. Mineral metabolism, mortality, and morbidity in maintenance hemodialysis. J Am Soc Nephrol 2004; 15(8): 2208-18.

15. Wang AY, Wang M, Woo J, et al. Cardiac valve calcification as an important predictor for all-cause mortality and cardiovascular mortality in long-term peritoneal dialysis patients: a prospective study. J Am Soc Nephrol 2003; 14: 159-68.

16. National Kidney Foundation DOQI: Clinical Pratical Guidelines For Bone Metabolism and Disease in Chronic Kidney Diseases. Am J Kidney Dis 2003; 42 (4), (Suppl 3).

17. Mazzaferro S, Cozzolino M, Marangella M, et al. Calcimimetics, phosphate binders, vitamin $\mathrm{D}$ and its analouges for treating secondary hyperparathyroidism in chronic kidney disease: guideline from the Italian Society of Nephrology. G Ital Nefrol 2007; 24 (Suppl 37): S107-24.

18. Kawata T, Imanishi Y, Kobayashi K, et al. Relationship between parathyroid calcium-sensing receptor expression and potency of the calcimimetic, cinacalcet, in suppressing parathyroid hormone secretion in an in vivo murine model of primary hyperparathyroidism. Eur J Endocrinol 2005; 153(4): 587-94.

19. Block G, Kevin JM, De Francisco ALM, et al. Cinacalcet for secondary hyperparathyroidism in patients receiving hemodialysis. N Engl J Med 2004; 350: 627-35.

20. Lindberg JS, Culleton B, Wong G, et al. Cinacalcet HCL, an oral calcimimetic agent for the treatment of secondary hyperparathyroidism in hemodialysis and peritoneal dialysis: A Randomized Double-Bind, Multicenter Study. J Am 
Soc Nephrol 2005; 16: 800-07.

21. Moe SM, Chertow GM, Coburn JW, et al. Achieving NKF$\mathrm{K} / \mathrm{DOQI} \mathrm{TM}$ bone metabolism and disease treatment goals with cinacalcet HCL. Kidney Int 2005; 67: 760-71.

22. Cunningham J, Danese M, Olson K, Klassen P, Chertow GM. Effect of the calcimimetic cinacalcet HCL on cardiovascular disease, fracture, and health-related quality of life in secondary hyperparathyroidism. Kidney Int 2005; 68 : 1793-800.

23. Szabo A, Merke J, Beier E, et al. $1,25(\mathrm{OH})_{2}$ vitamin $\mathrm{D}_{3}$ inhibits parathyroid cell proliferation in experimental uremia. Kidney Int 1989; 35: 1049-56.

24. Bellows CG, Reimers SM, Heersche JN. Expression of mRNAs for type I collagen, bone sialoprotein, osteocalcin and osteopontin at different stages of osteoblastic differentation and their regulation by 1,25 dihydroxyvitamin D. Cell Tissue Res 1999; 297: 249-59.

25. Sjoden G, Lindgren JU, DeLuca HF. Antirachitic activity of 1 alpha-hydroxyergocholecalciferol and 1 alpha-hydroxycholecalciferol in rats. J Nutr 1984; 114: 2043-6.

26. Brown AJ, Finch J, Grieff M, et al. The mechanism for the disparate actions of calcitriol and 22-oxacalcitriol in the intestine. Endocrinology 1993; 133: 1158-64.
27. Slatopolsky E, Cozzolino M, Finch JL. Differential effects of 19-nor-1,25- $(\mathrm{OH})_{2} \mathrm{D}_{2}$ and 1alpha-hydroxyvitamin $\mathrm{D}_{2}$ on calcium and phosphorum in normal and uremic rats. Kidney Int 2002; 62(4):1277-84.

28. Martin K, Gonzalez EA, Gellens M, et al. 19-Nor-1-alpha25-dihydroxyvitamin D2 (Paricalcitol) safely and effectively reduces the levels of intact parathyroid hormone in patients on hemodialysis. J Am Soc Nephrol 1998; 9(8):1427-32.

29. Sprague SM, Llach F, Amdahl M, et al. Paricalcitol versus calcitriol in the treatment of secondary hyperparathyroidism. Kidney Int 2003; 63(4): 1483-90.

30. Teng M, Wolf M, Lowrie E, et al. Survival of patients undergoing hemodialysis with paricalcitol or calcitriol. N Engl J Med 2003; 349: 446-56.

31. Teng M, Wolf M, Ofsthun MN, et al. Activated injectable vitamin $\mathrm{D}$ and hemodialysis survival: a historical cohort study. J Am Soc Nephrol 2005; 16: 1115-25.

32. Dobrez DG, Mathes A, Amdahl M, et al. Paricalcitoltreated patients experience improved hospitalization outcomes compared with calcitriol-treated patients in real-world clinical settings. Nephrol Dial Transplant 2004; 19(5):1174-81. 